\title{
Correction to: Design and multicriteria assessment of low-input cropping systems based on plant diversification in southwestern France
}

\author{
Catherine Bonnet ${ }^{1}$. Noémie Gaudio ${ }^{1}$ (i) - Lionel Alletto ${ }^{1}$. Didier Rafaillac ${ }^{1}$. Jacques-Eric Bergez ${ }^{1}$. \\ Philippe Debaeke $^{1} \cdot$ André Gavaland $^{2} \cdot$ Magali Willaume $^{1} \cdot$ Laurent Bedoussac $^{3}$ - Eric Justes ${ }^{1,4}$
}

Accepted: 15 October 2021 / Published online: 9 November 2021

(c) INRAE and Springer-Verlag France SAS, part of Springer Nature 2021

\section{Correction to: Agronomy for Sustainable Development} https://doi.org/10.1007/s13593-021-00719-7

In Table 2 of this article, some of the data in the second column headed 'Description and/or calculation - agro-economic output performance' were mistakenly listed under the first column headed 'Indicators - agro-economic output performance'. The correct version of Table 2 is published in this erratum.

The original article has been updated.

The original article can be found online at https://doi.org/10.1007/ s13593-021-00719-7.

Noémie Gaudio

noemie.gaudio@inrae.fr

1 AGIR, Univ. Toulouse, INRAE, Castanet-Tolosan, France

2 Unité Expérimentale Grandes Cultures Auzeville,

Univ. Toulouse, INRAE, Castanet-Tolosan, France

3 AGIR, Univ. Toulouse, ENSFEA, INRAE, Castanet-Tolosan,

France

4 Persyst Department, CIRAD, Montpellier, France 

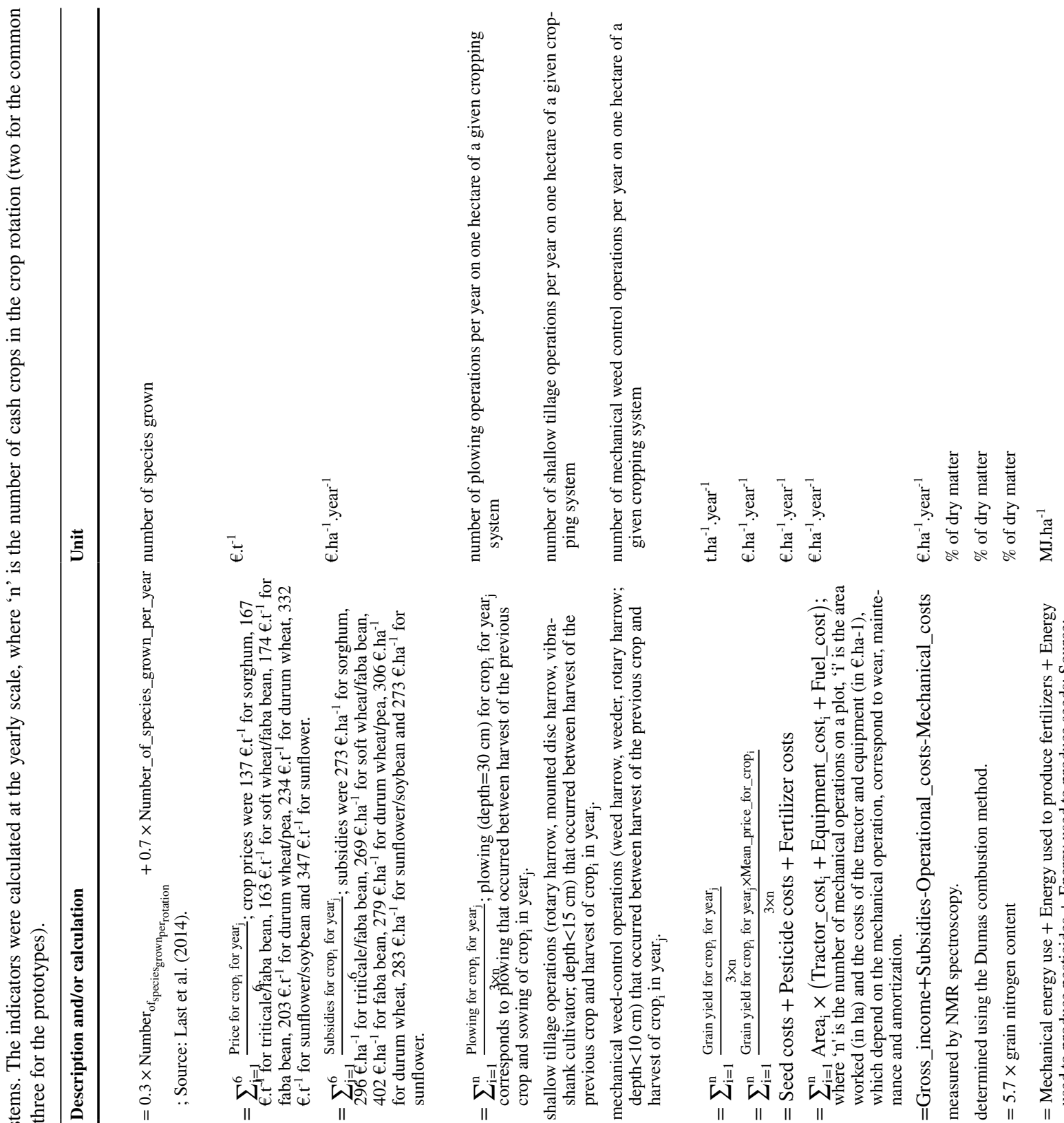

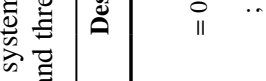
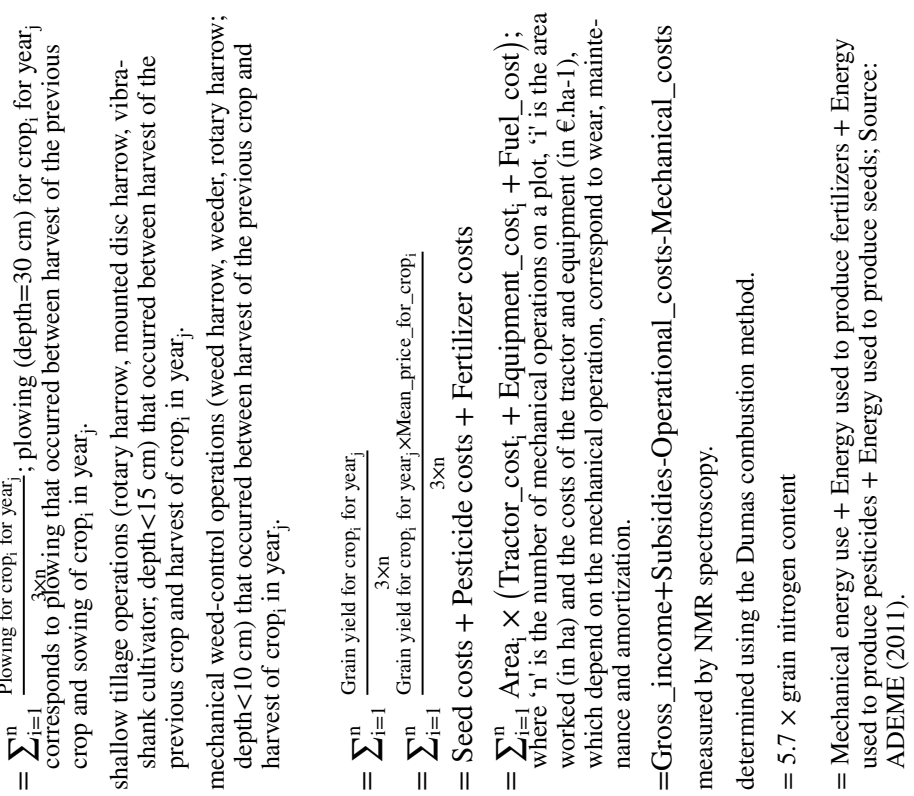

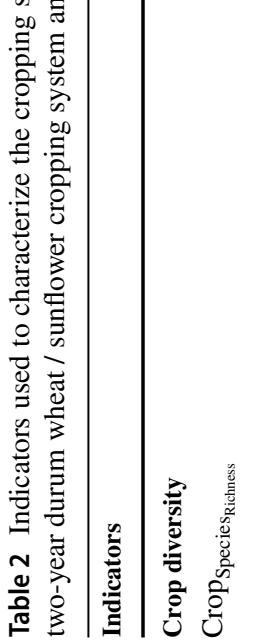
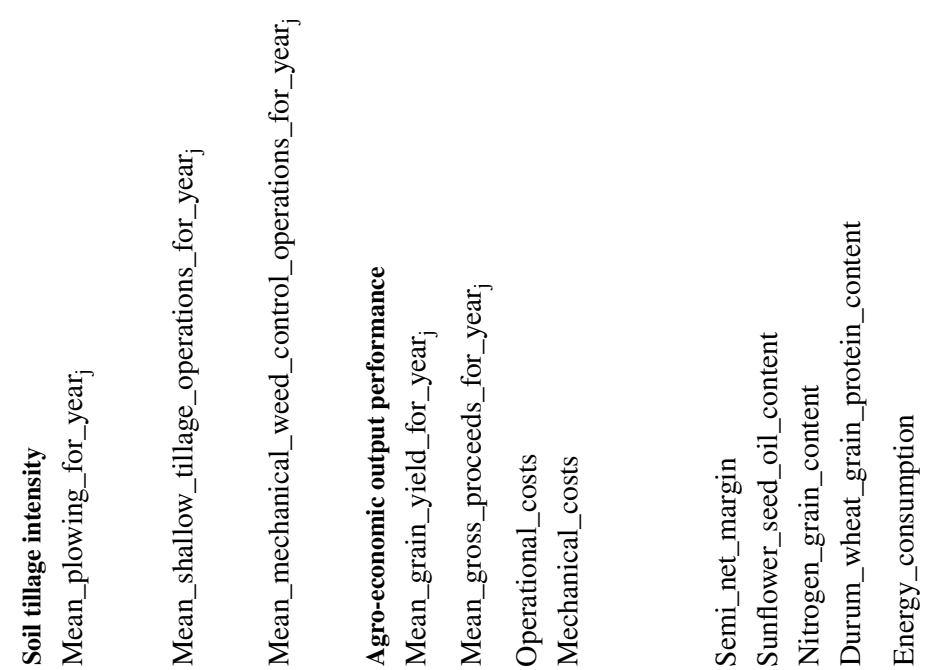


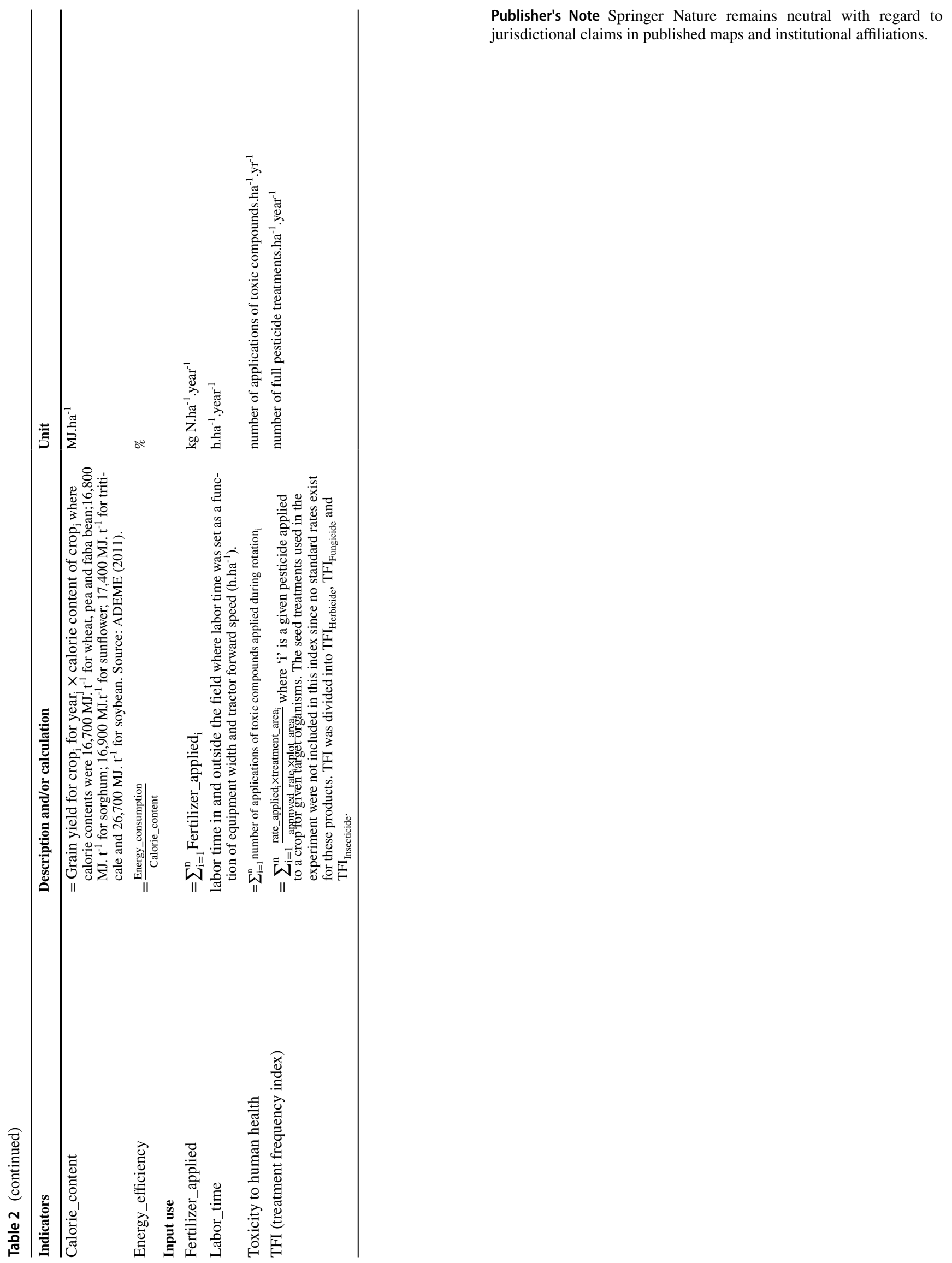

